\title{
Is There a Difference Between Minimal and Gross Extension into the Strap Muscles for the Risk of Recurrence in Papillary Thyroid Carcinomas?
}

\author{
Debora L.S. Danilovic, ${ }^{1,2}$ Luciana A. Castroneves, ${ }^{1}$ Claudia K. Suemoto, ${ }^{3}$ \\ Livia O. Elias, ${ }^{2}$ Ibere C. Soares, ${ }^{4}$ Rosalinda Y. Camargo, ${ }^{1}$ \\ Fernanda A. Correa, ${ }^{1}$ Ana O. Hoff, ${ }^{1}$ and Suemi Marui ${ }^{2}$
}

Background: The morbidity of papillary thyroid carcinoma (PTC) is primarily related to locoregional recurrences and distant metastases. The definition of minimal extrathyroidal extension (mETE) has been recently revised. The presence of mETE does not impact mortality and is generally not considered to be a predictor for the risk of recurrence. This study aimed at comparing the risk of recurrence and the response to therapy of PTC with mETE and gross extrathyroidal extension (ETE) into the strap muscles (gETE) with low- and intermediaterisk PTC without ETE (low risk w/o ETE and intermediate risk w/o ETE, respectively) to further characterize the impact of ETE on outcomes.

Methods: A total of 596 PTCs were analyzed according to the degree of ETE as well as other predictors of recurrence. Four groups of patients were compared, low risk w/o ETE $(n=251)$, intermediate risk w/o ETE $(n=89)$, mETE $(n=191)$, and gETE $(n=65)$, to determine the risk of recurrence and the response to treatment. Cox proportional hazards models were used to investigate associations between groups and disease-free survival (DFS).

Results: The risk of recurrence was 3\% in low risk w/o ETE PTC, 14\% in intermediate risk w/o ETE, $14 \%$ in mETE, and 25\% in gETE. The recurrence risk was increased by the presence of ETE (odds ratio [OR] $=2.86$, 95\% confidence interval [CI] $1.36-5.85, p=0.005)$ and lymph node metastases ( $\mathrm{OR}=2.44$ [95\% CI 1.25-4.76], $p=0.009$ ). Patients with low-risk carcinomas w/o ETE experienced longer DFS than those with mETE (hazard ratio $=0.08$ [95\% CI 0.02-0.28], $p<0.001$ ), but no significant difference was observed between intermediate risk w/o ETE, mETE, and gETE. In terms of the response to therapy, patients with gETE had higher rates of biochemical and/or structural incomplete responses within the first year of treatment (OR $=2.68$ [95\% CI 1.315.45], $p=0.007)$ and at the final follow-up evaluation ( $\mathrm{OR}=4.35$ [95\% CI 1.99-9.51], $p<0.001)$ compared with those with mETE. An analysis of the subgroups of microcarcinomas without lymph node metastases revealed no significant difference in DFS or the response to therapy between the low risk w/o ETE and mETE PTC groups.

Conclusions: The results of this study suggest that both mETE and gETE are independent risk factors for the risk of recurrence in PTC. Although gETE has a more pronounced impact on the recurrence risk and is associated with a worse response to therapy, mETE may not be associated with a low risk of recurrence. This observation suggests that patients with PTC and mETE may, in part, have an intermediate risk of recurrence and need to be followed accordingly.

Keywords: papillary thyroid carcinoma, extrathyroidal extension, lymph nodes metastases, recurrence, response to therapy

\footnotetext{
Departments of ${ }^{1}$ Endocrinology and ${ }^{4}$ Pathology, Instituto do Cancer do Estado de Sao Paulo, Faculdade de Medicina, Universidade de Sao Paulo, Sao Paulo, Brazil.

${ }^{2}$ Laboratorio de Endocrinologia Celular e Molecular (LIM25); ${ }^{3}$ Department of Geriatrics; Faculdade de Medicina, Hospital das Clinicas HCFMUSP, Universidade de Sao Paulo, Sao Paulo, Brazil.
} 


\section{Introduction}

D IFFERENTIATED THYROID CARCINOMAS (DTCs) usually have indolent behavior, and the increasing prevalence, particularly of papillary thyroid carcinomas (PTCs), is not associated with a higher mortality rate, which remains very low (1). However, several histological features of DTCs have been associated with poor outcomes. Histopathologic variants of PTCs, such as tall cell and columnar cell, and the hobnail variants, widely invasive follicular thyroid carcinoma, the presence of vascular invasion or lymph node metastases, and the presence of extrathyroidal extension (ETE) are associated with locoregional recurrences and distant metastases (2).

Extrathyroidal invasion occurs in up to $30 \%$ of DTCs (3), although extensive ETE to subcutaneous soft tissue, the trachea, larynx, esophagus, and laryngeal nerves is observed in only $\sim 5 \%$ (4). Most recently, the American Joint Commission on Cancer (AJCC) concluded that minimal ETE (mETE), detected only on histological examination, had no impact on mortality and proposed that only gross ETE, which corresponds to the macroscopic invasion of a tumor apparent on imaging or during surgery, is clinically relevant and does impact the staging of these tumors. In the updated 8th version of the AJCC staging system, macroscopic ETE into strap muscle is classified as T3b; macroscopic extrathyroidal invasion of subcutaneous soft tissue, the larynx, trachea, esophagus, or recurrent laryngeal nerve is considered as T4a; and invasion of the prevertebral fascia, encasing of the carotid artery, or mediastinal vessels is classified as T4b (5).

Concerning recurrence, the 2015 American Thyroid Association (ATA) guidelines still consider the presence of mETE as a feature for an intermediate risk of recurrence (2). A recent meta-analysis revealed an overall increased risk of recurrence associated with mETE (3), with an odds ratio (OR) of 1.76, but the eligible studies had, in part, divergent findings. Four $(6-9)$ of six $(10,11)$ studies evaluating DTCs with mETE without lymph node metastases found no statistically significant increase in the risk of recurrence. On the other hand, studies that included patients with and without lymph node metastases suggested that mETE would be an additional risk factor for recurrence (3). The aforementioned studies failed to include vascular invasion or histological features, including unfavorable variants, which are well-established predictors for a higher risk of recurrence, in the analyses to determine the prognostic role of ETE. Thus, the actual importance of mETE remains somewhat controversial.

Whether gross extrathyroidal extension into the strap muscles (gETE) compared with extension restricted to perithyroidal soft tissue is associated with compromised outcomes of patients with DTC has also been debated. Amit et al. evaluated the impact of gross strap muscle invasion for DTCs $\leq 4 \mathrm{~cm}$ on survival and recurrence, compared to no ETE and mETE into perithyroidal soft tissue only. Overall and diseasespecific survival rates were not significantly different between the three groups. On multivariate analysis, neither mETE nor gETE was associated with worse disease-free survival (DFS), but it was noteworthy that only mETE was associated with worse locoregional recurrence-free survival compared with no ETE (hazard ratio [HR] 2.02, 95\% confidence interval [CI] 1.06-3.94) (12).
With the goal to shed some light onto these controversial views, the aims of this study were (1) to compare the risk of recurrence and the response to therapy of PTCs with different degrees of extrathyroidal invasion (i.e., minimal and gross ETE into the strap muscles) with low- and intermediate-risk PTCs without ETE and (2) to establish the impact of ETE, independent of other risk factors, on the risk of recurrence and the response to therapy.

\section{Materials and Methods}

\section{Patients}

We prospectively followed 786 patients with DTC, who were evaluated in the Endocrinology Department of the Instituto do Cancer do Estado de Sao Paulo (ICESP) from 2012 to 2018. Clinical characteristics, histopathological features, laboratory, and imaging examinations were collected. All patients were clinically evaluated every 6-12 months or more frequently if necessary and underwent measurements of serum thyroglobulin (sTg), antithyroglobulin antibodies (TgAbs), and TSH concentrations and evaluation with neck ultrasonography (US). Complementary examinations, including fineneedle aspiration biopsies (FNABs), were performed to localize structural persistent disease if suspicious lymph nodes on US were identified; chest or neck computed tomography (CT) scans, diagnostic ${ }^{131} \mathrm{I}$ whole body scans, or positive emission tomography with fluorodeoxyglucose $\left({ }^{18}\right.$ FDG-PET)/CT scans were performed in patients with elevated sTg or rising $\mathrm{TgAb}$ concentrations.

After approval by the Institutional Ethics Committee of University of Sao Paulo, patients with PTC followed for more than one year were selected for analysis. Age, sex, and histological data (histological variant, tumor size, multifocality, lymphovascular invasion, lymph node metastases, and the presence of ETE) were collected by retrospective chart review. Subjects were classified according to the 8th AJCC edition-tumor, lymph node involvement, and metastasis (TNM) - classification (5) and according to the 2015 ATA classifications for the risk of recurrence and the response to therapy (2).

Persistent disease was defined as structural or functional (radioactive iodine [RAI] scan) evidence of metastases (locoregional or distant metastases) diagnosed at less than six months after initial therapy by neck US, ${ }^{131}$ I whole body scanning, or ${ }^{18}$ FDG-PET/CT. Recurrence was defined as an identifiable disease only after six months after initial therapy as long as there was no uptake outside the thyroid bed on the initial post-therapy whole body scan. New locoregional and distant metastases were diagnosed and confirmed with an FNAB of suspicious lymph node or biopsy of suspicious lesion or functional evidence (RAI scan, ${ }^{18}$ FDG-PET) after persistent abnormal sTg or rising $\mathrm{TgAb}$ levels. The response to therapy was evaluated according to the 2015 ATA classification (2) - excellent, indeterminate, biochemical incomplete, or structural incomplete responses-within the first year of initial treatment and at the final follow-up visit. The follow-up period was defined as the time from initial treatment until the last medical visit.

Follicular carcinomas and Hürthle cell carcinomas; PTC with extrathyroidal invasion of subcutaneous soft tissue, the larynx, trachea, esophagus, or recurrent laryngeal nerve (T4a) or invasion of the prevertebral fascia, encasing the 
carotid artery, or mediastinal vessels (T4b); the presence of distant metastases at diagnosis or up to 6 months from initial therapy; and patients followed for $<12$ months from initial therapy were excluded from the analysis.

Most patients (99\%) underwent total thyroidectomy as the initial surgery or completion thyroidectomy after diagnostic lobectomy. Neck dissections were performed when lymph node metastases had been diagnosed preoperatively or intraoperatively ( $18 \%$ central, $11 \%$ central and lateral, and $0.7 \%$ lateral neck dissections). Most patients $(73.5 \%)$ received RAI ablation therapy after surgery. Patients were divided into four groups based on the 2015 ATA classification for recurrence risk (2) and the presence and degree of ETE of PTC as follows:

1. Low risk without ETE (low risk w/o ETE), if the PTC was classified as ATA low risk of recurrence, which corresponds to an intrathyroidal tumor, without local metastases and all of the macroscopic tumor was resected, favorable histology (classic or follicular variants), no vascular invasion, and clinical $\mathrm{N} 0$ or $\leq 5$ pathologic N1 micrometastases $(<0.2 \mathrm{~cm})$.

2. Intermediate risk w/o ETE (intermediate risk w/o ETE), if the PTC without ETE was classified as ATA intermediate risk, which includes the presence of lymph node metastases (clinical N1 or $>5$ pathologic $\mathrm{N} 1$ with all metastatic lymph nodes $<3 \mathrm{~cm}$ without extranodal extension), an unfavorable variant (all, but classic or the follicular variant), and vascular invasion.

3. mETE, if, on pathology report, extrathyroidal invasion was restricted to perithyroidal soft tissues, including microscopic strap muscle invasion.

4. gETE, if, on pathology report, strap muscle invasion was present and the macroscopic invasion was observed on preoperative imaging and/or at the time of surgery (5).

\section{Statistical analysis}

Categorical variables are presented as absolute and relative frequencies. Differences between PTC subgroups were evaluated by the chi-square test and Fisher's exact test when appropriate. Continuous variables are presented as the mean and standard deviation or the median and range. Differences among the studied subgroups were determined using Student's $t$-test, the Mann-Whitney $U$ test, Kruskal-Wallis test, or analysis of variance (ANOVA) test, with Bonferroni correction, when indicated.

Univariate and multivariate logistic regression models were used to investigate the association of sociodemographic and clinical variables (age $\geq 55$ years, sex, tumor size $>4 \mathrm{~cm}$, unfavorable histology, multifocality, lymphovascular invasion, ETE, lymph node metastases, and radioiodine therapy) with recurrence risk. A multivariate logistic regression model was used to identify independent prognostic factors of recurrence and to compare the response to therapy between groups, adjusting for age, sex, tumor size, unfavorable histology, multifocality, lymphovascular invasion, lymph node metastases, and radioiodine therapy.

The Kaplan-Meier method was used to estimate survival factors for DFS among groups. Cox proportional hazards (PH) models were adopted for the multivariate analysis, ad- justing for age, sex, tumor size, unfavorable histology, multifocality, lymphovascular invasion, lymph node metastases, and radioiodine therapy. The $\mathrm{PH}$ assumption was checked by graphical inspection of both Kaplan-Meier curves and loglog curves of each group.

Data were analyzed using IBM SPSS Statistics for Windows, version 24.0 (IBM Corp., Armonk, NY), Stata 15 (StataCorp 2017, College Station, TX), and two-tailed tests, and the alpha level was set at the $5 \%$ level.

\section{Results \\ Clinical characteristics}

Five hundred ninety-six patients with PTC were selected for analysis. Eighty-eight percent were female, with a mean age of $49.7 \pm 13.6$ years. Most patients had T1 tumors $(66 \%)$. Lymph node metastases (N1) were present in 171 patients (29\%): 160 patients were diagnosed with metastases at surgery, and 11 were found to have metastases on whole body scans with SPECT/CT before and/or after RAI ablation ( 6 patients with mETE, 4 with intermediate risk w/o ETE, and 1 with gETE). Eighty-seven percent were classified with the AJCC system as stage I and $13 \%$ as stage II. The median follow-up period was 44.4 months (range 12-322 months). The clinical and pathological features of each group are shown in Table 1.

mETE was reported in 191 carcinomas, and gETE was observed in 65 carcinomas (Table 1). The comparison of clinical and pathologic features revealed that tumors with gETE were larger than those with $\operatorname{mETE}(p<0.001)$, with $20 \%$ of tumors associated with gETE larger than $4 \mathrm{~cm}$ compared with $7 \%$ with mETE $(p=0.003)$. Neck dissections were more frequently performed in patients with gETE $(58.5 \%)$ than mETE $(42.4 \%, p=0.025)$. Although a higher rate of lymphovascular invasion was observed in tumors with gETE $(p<0.001)$, there was no statistically significant difference in the frequency of lymph node metastases. Compromised surgical margins were similar in carcinomas with mETE and gETE $(32 \%, p=0.98)$.

The mETE and gETE groups were compared with the low risk w/o ETE and intermediate risk w/o ETE groups (Table 1). Patients with mETE and gETE were significantly older than intermediate-risk patients w/o ETE $(p=0.009)$, but the mean age did not differ from that of low-risk patients w/o ETE. Low risk w/o ETE carcinomas had the smallest mean tumor size $(p<0.001)$, and carcinomas with mETE were smaller than intermediate risk w/o ETE carcinomas $(p=0.006)$. Unfavorable variants of PTC were more frequent in the intermediate-risk group w/o ETE (22.5\%), particularly when compared with patients with mETE tumors $(p=0.003)$. Neck dissections were more frequently performed in the mETE, gETE, and intermediate risk w/o ETE groups than in the low risk w/o ETE group $(p<0.001)$, but there was no statistically significant difference between patients with mETE or gETE and those with intermediate risk w/o ETE. The rates of lymph node metastases did not differ between carcinomas with mETE (13.6\%), gETE (23.1\%), and intermediate risk w/o ETE (11.2\%). Radioiodine therapy was more frequently used in the mETE, gETE, and intermediate risk w/o ETE groups than in the low risk w/o ETE group $(p<0.001)$. 
Table 1. Clinical and Pathological Characteristics, Risk of Recurrence, and Response to Therapy According to the Presence or Absence of Extrathyroidal Extension

\begin{tabular}{|c|c|c|c|c|c|}
\hline & $\begin{array}{c}\text { Low risk w/o ETE } \\
(\mathrm{n}=251)\end{array}$ & $\begin{array}{l}\text { Intermediate risk w/o ETE } \\
\qquad(\mathrm{n}=89)\end{array}$ & $\begin{array}{c}\text { mETE } \\
(\mathrm{n}=191)\end{array}$ & $g E T E(\mathrm{n}=65)$ & $\mathrm{p}^{\mathrm{a}}$ \\
\hline Female, $n(\%)$ & $231(92)$ & $76(85.4)$ & 166 (86.9) & $54(83.1)$ & 0.101 \\
\hline Mean age (years) & $50.8 \pm 12$ & $44.9 \pm 15.8$ & $50.0 \pm 13.7$ & $51.6 \pm 14.5$ & 0.002 \\
\hline \multicolumn{5}{|l|}{ Neck dissection (\%) } & \multirow{3}{*}{$<0.001$} \\
\hline Central & $10(4.0)$ & $31(34.8)$ & 45 (23.6) & $20(30.8)$ & \\
\hline Lateral $^{\mathrm{b}}$ & $1(0.4)$ & $15(16.9)$ & $36(18.8)$ & $18(27.7)$ & \\
\hline Mean tumor size $(\mathrm{mm})$ & $14.9 \pm 16.4$ & $24.1 \pm 18.9$ & $17.3 \pm 13.4$ & $28.0 \pm 16.0$ & $<0.001$ \\
\hline Unfavorable variant (\%) & - & $20(22.5)$ & $16(8.4)$ & $11(16.9)$ & $<0.001$ \\
\hline Multifocality (\%) & $107(43)$ & $57(64.8)$ & $121(63.4)$ & $35(54.7)$ & $<0.001$ \\
\hline Lymphovascular invasion (\%) & 0 & $29(33.7)$ & $42(22)$ & $31(49.2)$ & $<0.001$ \\
\hline \multicolumn{5}{|l|}{ Tumor stage } & \multirow[t]{4}{*}{$<0.001$} \\
\hline T1 & $200(79.7)$ & $52(59.1)$ & $141(73.8)$ & - & \\
\hline $\mathrm{T} 2$ & 37 (14.7) & $22(25)$ & $35(18.3)$ & - & \\
\hline T3 & $14(5.6)$ & $14(15.9)$ & $15(7.9)$ & $65(100)$ & \\
\hline \multicolumn{5}{|l|}{ Lymph node metastases $(\%)^{\mathrm{c}}$} & \multirow{5}{*}{$<0.001$} \\
\hline $\mathrm{Nx}$ & $148(59)$ & $21(23.6)$ & $54(28.3)$ & $18(27.7)$ & \\
\hline No & $103(41)$ & $19(21.3)$ & $62(27.2)$ & $10(15.4)$ & \\
\hline N1a & - & $32(36.0)$ & $45(23.6)$ & $19(29.2)$ & \\
\hline N1b & - & $17(19.1)$ & $40(20.9)$ & $18(27.7)$ & \\
\hline Radioiodine therapy (\%) & $102(40.6)$ & $81(91)$ & $186(97.4)$ & $64(98.5)$ & $<0.001$ \\
\hline \multicolumn{5}{|l|}{ Dose (mCi) } & \\
\hline Recurrence $(\%)$ & $8(3.2)$ & $12(13.5)$ & $26(13.6)$ & $16(24.6)$ & \multirow[t]{6}{*}{$<0.001$} \\
\hline Lymph node metastases (\%) & $7(2.8)$ & $10(11.2)$ & $26(13.6)$ & $15(23.1)$ & \\
\hline Central & $3(1.2)$ & $1(1.1)$ & $8(4.2)$ & $3(4.6)$ & \\
\hline Lateral & $3(1.2)$ & $5(5.6)$ & $10(5.2)$ & $3(4.6)$ & \\
\hline Central and lateral & $1(0.4)$ & $4(4.5)$ & $8(4.2)$ & $9(13.8)$ & \\
\hline Distant metastases (\%) & $1(0.4)$ & $3(3.4)$ & $5(2.6)$ & $10(15.4)$ & \\
\hline \multicolumn{5}{|l|}{ Response first year $(\%)^{\mathrm{d}, \mathrm{e}}$} & \multirow[t]{5}{*}{$<0.001$} \\
\hline Excellent & $167(67.9)$ & $58(68.2)$ & $125(66.1)$ & $24(39.3)$ & \\
\hline Indeterminate & $71(28.9)$ & $16(18.8)$ & $31(16.4)$ & $11(18)$ & \\
\hline Biochemical incomplete & $4(1.6)$ & $6(7.1)$ & $21(11.1)$ & $18(29.5)$ & \\
\hline Structural incomplete & $4(1.6)$ & $5(5.9)$ & $12(6.3)$ & $8(13.1)$ & \\
\hline \multicolumn{5}{|l|}{ Response final evaluation (\%) } & \multirow[t]{5}{*}{$<0.001$} \\
\hline Excellent & $181(73)$ & $59(67)$ & $132(70.6)$ & 27 (42.9) & \\
\hline Indeterminate & $62(25)$ & $21(23.9)$ & $36(19.3)$ & $13(20.6)$ & \\
\hline Biochemical incomplete & $4(1.6)$ & $5(5.7)$ & $12(6.4)$ & $15(23.8)$ & \\
\hline Structural incomplete & $1(0.4)$ & $3(3.4)$ & $7(3.7)$ & $8(12.7)$ & \\
\hline Cancer-related mortality & 0 & $1(1.1)$ & $1(0.5)$ & $1(1.5)$ & 0.34 \\
\hline $\begin{array}{l}\text { Median follow-up, months } \\
\text { (min-max) }\end{array}$ & $48(12-322)$ & $43.2(12-217)$ & $39.6(12-142)$ & $43.2(12-260)$ & 0.001 \\
\hline
\end{tabular}

${ }^{\mathrm{a}} p$ refers to comparison among the four groups.

${ }^{\mathrm{b}}$ Lateral neck dissection corresponds to lateral with and without central neck dissection.

${ }^{\mathrm{c}} \mathrm{Nx} / \mathrm{N} 0$ compared with $\mathrm{N} 1 \mathrm{a} / \mathrm{N} 1 \mathrm{~b}$ at surgery or on RAI scans.

${ }^{\mathrm{d}}$ Excellent/indeterminate compared with biochemical incomplete/structural incomplete responses.

${ }^{\mathrm{e}}$ Percentages refer to patients whose responses to therapy were available.

ATA, American Thyroid Association; ETE, extrathyroidal extension; gETE, gross extrathyroidal extension into the strap muscles; intermediate risk w/o ETE, ATA intermediate risk without ETE; low risk w/o ETE, ATA low risk of recurrence without ETE; mETE, minimal ETE; RAI, radioactive iodine.

\section{Risk of recurrence}

Only three patients had persistent disease: two had carcinomas with mETE and lymph node metastases at presentation and one had an intermediate-risk carcinoma w/o ETE. Disease recurred in 62 patients: 43 presented only lymph node metastases, 3 only distant metastases, and 16 both lymph node and distant metastases. Distant metastases oc- curred in the lungs (63\%), bones (5\%), and multiple sites $(32 \%)$. After exclusion of persistent disease, the risk of recurrence was evaluated according to clinical and pathologic features (Table 2). On univariate analysis, age, tumor size, lymphovascular invasion, ETE, lymph node metastases, and radioiodine therapy were associated with an increased risk of recurrence. However, in the multivariate analysis, only ETE $(\mathrm{OR}=2.83$ [95\% CI 1.36-5.85], $p=0.005)$ and lymph node 
Table 2. Clinical and Pathological Characteristics Associated with Recurrence in 593 Patients With Papillary Thyroid Carcinoma

\begin{tabular}{|c|c|c|c|c|c|c|}
\hline & \multicolumn{3}{|c|}{ Univariate analysis } & \multicolumn{3}{|c|}{ Multivariate analysis } \\
\hline & $O R$ & $95 \% C I$ & $\mathrm{p}$ & $O R$ & $95 \% C I$ & $\mathrm{p}$ \\
\hline Male & 1.83 & $0.90-3.71$ & 0.09 & 1.66 & $0.77-3.56$ & 0.19 \\
\hline Age & 1.02 & $1.01-1.05$ & 0.015 & 1.02 & $1.00-1.05$ & 0.08 \\
\hline Tumor size & 0.98 & $0.97-1.00$ & 0.015 & 0.99 & $0.97-1.01$ & 0.19 \\
\hline Unfavorable histology & 1.02 & $0.39-2.69$ & 0.97 & 1.02 & $0.37-2.85$ & 0.97 \\
\hline Multifocality & 1.06 & $0.62-1.83$ & 0.83 & 0.86 & $0.48-1.56$ & 0.63 \\
\hline Lymphovascular invasion & 2.19 & $1.19-4.04$ & 0.012 & 0.86 & $0.41-1.78$ & 0.67 \\
\hline ETE & 3.71 & $2.06-6.68$ & $<0.001$ & 2.83 & $1.36-5.85$ & 0.005 \\
\hline Lymph node metastases & 3.88 & $2.26-6.65$ & $<0.001$ & 2.44 & $1.25-4.76$ & 0.009 \\
\hline Radioiodine therapy & 2.41 & $1.16-5.0$ & 0.019 & 0.87 & $0.34-2.22$ & 0.77 \\
\hline
\end{tabular}

CI, confidence interval; OR, odds ratio.

metastases $(\mathrm{OR}=2.44$ [95\% CI 1.25-4.76], $p=0.009)$ remained risk factors for recurrence.

DFS of mETE, gETE, low risk w/o ETE, and intermediate risk w/o ETE groups is shown in Kaplan-Meier plots (Fig. 1A). Compared with patients with mETE, patients with low risk w/o ETE tumors had longer DFS (median 46.8 months in low risk w/o ETE [6-196] vs. 30 months in mETE [6-112], HR $=0.08$ [95\% CI 0.02-0.28], $p<0.001$ ). However, DFS in the intermediate risk w/o ETE [median 37.6 months (5-138)] and gETE [median 31.2 months (7-226)] groups did not differ significantly from that in the mETE group (Table 3). The rates of lymph node metastases diagnosed during the follow-up of patients with mETE, gETE, or intermediate risk w/o ETE PTCs were not statistically significantly different, but distant metastases were more frequently diagnosed in patients with gETE than those with mETE $(p<0.001)$ or intermediate risk w/o ETE tumors $(p=0.016)$ (Table 1).

\section{Response to therapy}

Biochemical and/or structural incomplete responses after the first year of treatment and at the final follow-up evaluation of all groups are illustrated in Figure 2A. Compared with the
mETE group, the low risk w/o ETE group had a lower frequency of incomplete responses within the first year of therapy $(\mathrm{OR}=0.18$ [95\% CI 0.05-0.58], $p=0.004)$, but the difference was not significant during follow-up $(\mathrm{OR}=0.32$ [95\% CI 0.08-1.33], $p=0.12$ ). The rates of biochemical and/or structural incomplete responses to therapy within the first year and at the final evaluation were not significantly different between subjects with mETE and intermediate risk w/o ETE tumors.

However, despite the absence of a significant difference in DFS, patients with gETE had higher rates of biochemical and/or structural incomplete responses compared with patients with mETE tumors within the first year of therapy $(\mathrm{OR}=2.68$ [95\% CI 1.31-5.45], $p=0.007)$ and at the final follow-up evaluation $(\mathrm{OR}=4.35 \quad[95 \%$ CI 1.99-9.51], $p<0.001)$.

\section{ETE and lymph node metastases}

The prognostic influence of lymph node metastases on outcomes of patients with mETE and gETE was evaluated (Table 4). DFS according to the degree of ETE and lymph node status (N0 and N1) is illustrated in Kaplan-Meier plots (Fig. 1B). The median DFS times were as follows: mETE+NO
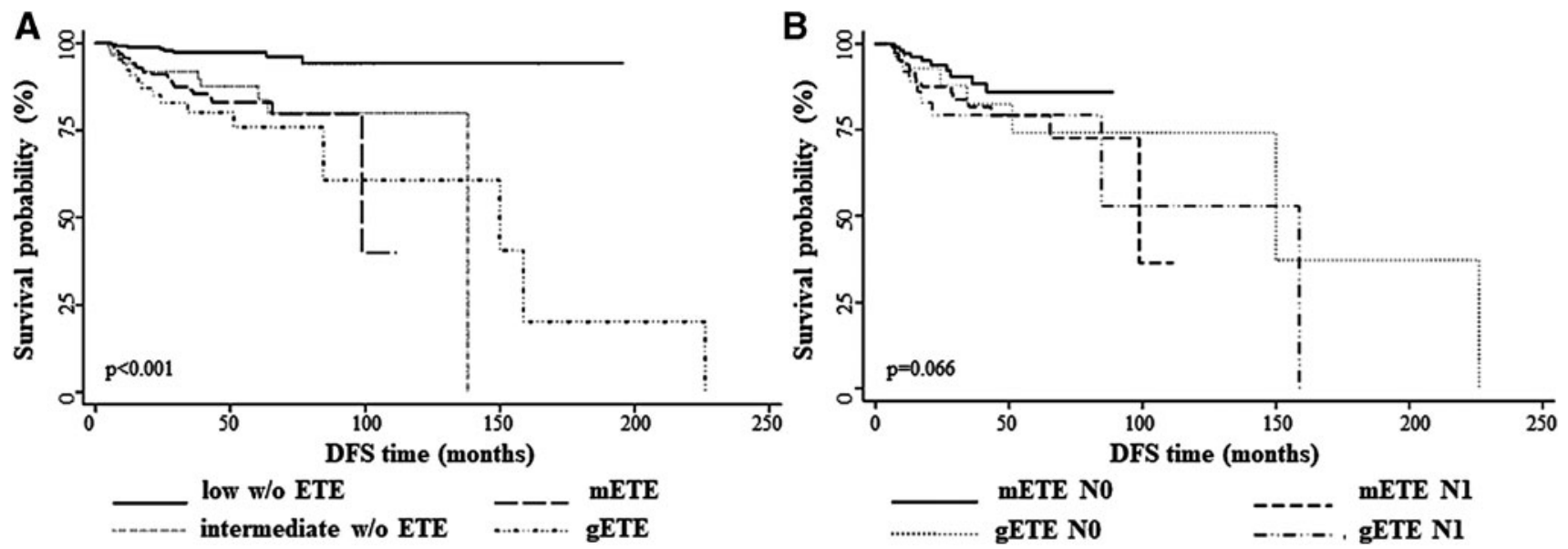

FIG. 1. DFS of papillary thyroid carcinomas. (A) DFS of carcinomas with mETE, gETE, low risk w/o ETE, and intermediate risk w/o ETE. (B) DFS of mETE and gETE carcinomas according to the presence (N1) or absence (N0) of lymph node metastases. DFS, disease-free survival; ETE, extrathyroidal extension; gETE, gross extrathyroidal extension into the strap muscles; mETE, minimal ETE. 
Table 3. Cox Proportional Hazards Regression Analysis for Variables Associated with Disease-Free Survival in Papillary Thyroid Carcinomas

\begin{tabular}{lcccc}
\hline & Crude HR [95\% CI] & $\mathrm{p}$ & Adjusted HR [95\% CI $]^{\mathrm{a}}$ & $\mathrm{p}$ \\
\hline mETE & Reference & & Reference & \\
Low risk w/o ETE & $0.17[0.08-0.37]$ & $<0.001$ & $0.08[0.02-0.28]$ & $<0.001$ \\
Intermediate risk w/o ETE & $0.88[0.44-1.75]$ & 0.71 & $0.70[0.34-1.47]$ & 0.35 \\
gETE & $1.30[0.66-2.54]$ & 0.45 & $1.30[0.63-2.71]$ & 0.48 \\
\hline
\end{tabular}

HR, hazard ratio.

${ }^{a}$ Cox proportional hazards models adjusted for age, sex, tumor size, unfavorable variant, multifocality, lymphovascular invasion, lymph node metastases, and radioiodine therapy.

28.5 months (6-89), mETE+N1 33.6 months (7-112), gETE+N0 39.6 months (7-226), and gETE+N1 28.8 months (7-159). The comparison of DFS, adjusted for age, sex, tumor size, unfavorable histology, multifocality, lymphovascular invasion, surgical margins, and radioiodine therapy, revealed no significant differences (Table 4).

The rates of biochemical and/or structural incomplete responses of mETE+N0, mETE+N1, gETE+N0, and gETE+N1 tumors within the first year of treatment and at the final follow-up evaluation are illustrated in Figure 2B. Compared with mETE+N0, only gETE+N1 tumors were associated with a significantly higher frequency of incomplete responses within the first year of therapy $(\mathrm{OR}=5.80$ [95\% CI $1.95-$ 17.20], $p=0.002)$ and at the final evaluation $(\mathrm{OR}=9.32[95 \%$ CI 2.53-34.41], $p=0.001$ ).

\section{Microcarcinomas}

The outcomes of patients with microcarcinomas $(\leq 1 \mathrm{~cm})$ without lymph node metastases (N0) at diagnosis were also analyzed. This subgroup comprised 136 low risk w/o ETE

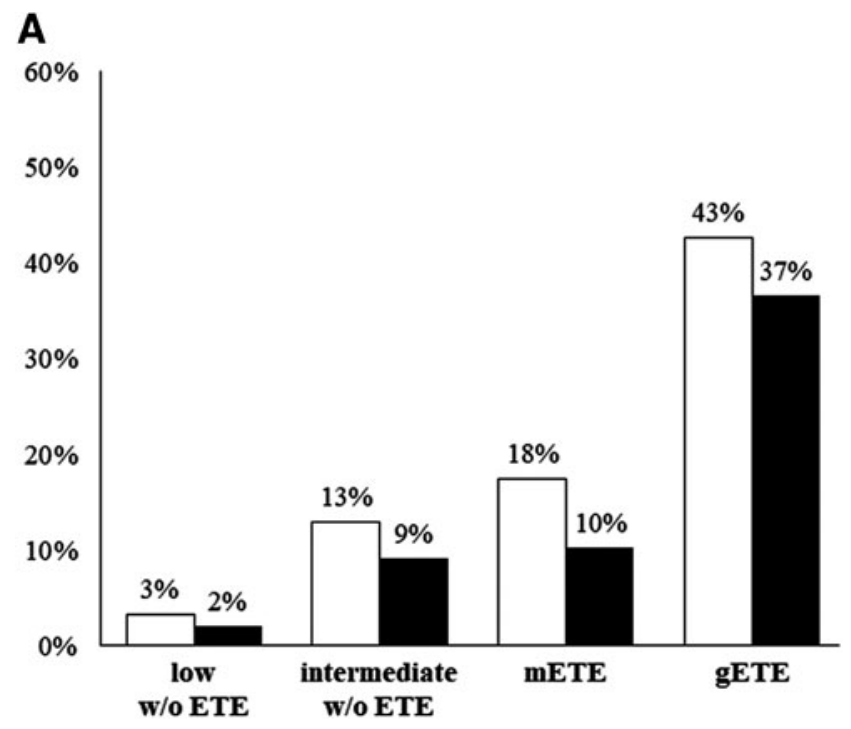

Biochemical and structural incomplete responses within first year

Biochemical and structural incomplete responses at final evaluation and 7 intermediate risk w/o ETE carcinomas, 44 carcinomas with mETE, and 2 with gETE. Recurrence occurred in only $3.7 \%$ of these tumors (4 low risk w/o ETE, no intermediate risk w/o ETE, 3 carcinomas with mETE, and 1 with gETE).

Due to the low number of subjects in the subgroups with gETE and intermediate risk w/o ETE, the outcomes were analyzed only for low risk w/o ETE carcinomas and microcarcinomas with mETE. The median DFS was 53.4 months (8-196) in the low risk w/o ETE group and 34.8 months (9-83) in the mETE N0 group, which was not significantly different after adjusting for age, sex, tumor size, unfavorable histology, multifocality, lymphovascular invasion, and radioiodine therapy $(p=0.71)$. Biochemical and/or structural incomplete responses occurred in $11.4 \%$ of mETE N0 tumors and $3 \%$ of low risk w/o ETE carcinomas within the first year and in $2.3 \%$ of mETE N0 and $1.5 \%$ of low risk w/o ETE tumors at the final evaluation. The comparison between the low risk w/o ETE and mETE N0 subgroups revealed no significant difference after adjusting for clinical pathological features, either within the first year $(p=0.28)$ or at the final evaluation $(p=1.0)$.

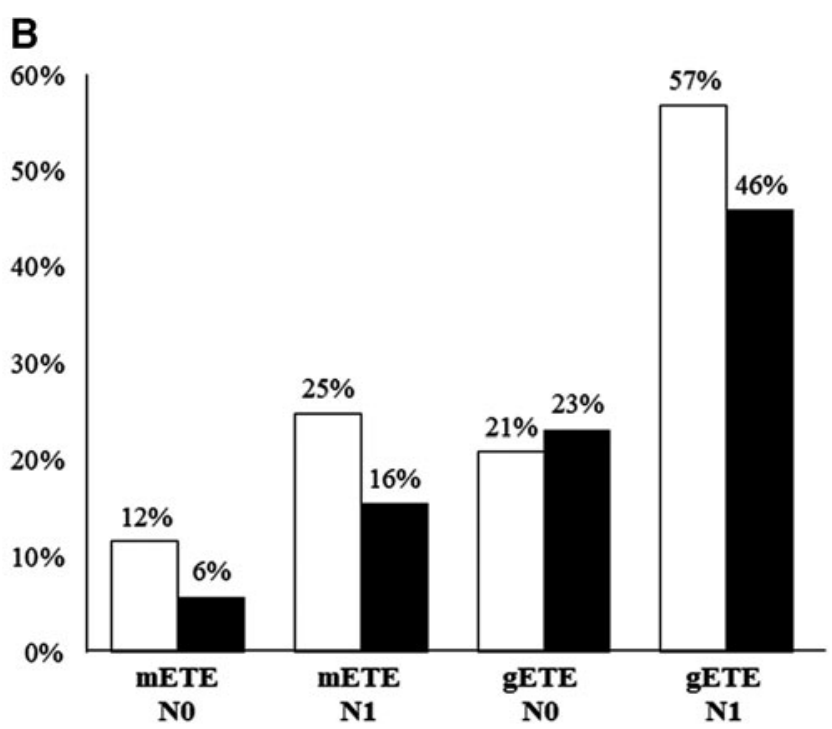

Biochemical and structural incomplete responses within first year

Biochemical and structural incomplete responses at final evaluation

FIG. 2. Response to therapy of papillary thyroid carcinomas within the first year of therapy and at final medical evaluation. (A) Biochemical and structural incomplete responses of carcinomas with mETE, gETE, low risk w/o ETE, and intermediate risk w/o ETE. (B) Biochemical and structural incomplete responses of mETE and gETE carcinomas according to the presence $(\mathrm{N} 1)$ or absence (N0) of lymph node metastases. 
Table 4. Cox Proportional Hazards Regression ANAlysis FOR VARIABles ASSOCIATED with Disease-Free Survival in Papillary Thyroid Carcinomas With EXtrathyroidal Extension

\begin{tabular}{lcccc}
\hline & $\begin{array}{c}\text { Crude HR } \\
{[95 \% C I]}\end{array}$ & $\mathrm{p}$ & $\begin{array}{c}\text { Adjusted HR } \\
{[95 \% C I]^{\mathrm{a}}}\end{array}$ & $\mathrm{p}$ \\
\hline mETEN0 & Reference & & Reference & \\
mETE N1 & $1.94[0.88-4.29]$ & 0.10 & $1.54[0.65-3.68]$ & 0.33 \\
gETE N0 & $1.59[0.56-4.57]$ & 0.39 & $1.61[0.52-4.96]$ & 0.41 \\
gETE N1 & $2.17[0.86-5.48]$ & 0.10 & $1.91[0.61-5.99]$ & 0.27 \\
\hline
\end{tabular}

${ }^{\mathrm{a}} \mathrm{Cox}$ proportional hazards models adjusted for age, sex, tumor size, unfavorable variant, multifocality, lymphovascular invasion, surgical margin, and radioiodine therapy.

N0, absence of lymph node metastases; N1, presence of lymph node metastases.

\section{Discussion}

The presence of ETE has been implicated in the poor prognosis of DTCs (2). However, after recent modifications in the 8th AJCC staging system (5), in which mETE was excluded from the T3 tumor classification, several reports have reviewed the role of $\mathrm{mETE}$ in DTC outcomes $(3,12-14)$.

The diagnosis of mETE is usually subjective and varies among pathologists. The thyroid is covered by discontinuous fibroadipose connective tissue, the internal thyroid capsule, with nonhomogeneous thickness and composition. Moreover, bundles of skeletal muscle are normally located within the parenchyma, particularly in the isthmus (15). For these reasons, the diagnosis of mETE can be challenging. Concordance among pathologists about the presence of ETE is higher when it is assessed using the relationship between carcinoma and skeletal muscle, but low interobserver agreement occurs when only adipose tissue, vasculature, or nerves, rather than skeletal muscle, are considered (16). On the other hand, gETE usually manifests itself by the adherence of the gland to strap muscles or other surrounding structures. If strap muscles are adherent to the gland, they can be resected en bloc to improve surgical results (15). Ito et al. previously compared patients with PTC without ETE with patients with PTC with massive ETE (extension into subcutaneous soft tissue and surrounding structures) and patients with PTC with minimal invasion of perithyroidal soft tissues and strap muscles (17). Higher rates of recurrence were observed only in tumors with massive ETE. Other studies have demonstrated that macroscopic ETE had a 6.0-fold increased risk of recurrence and significantly lower DFS when compared with PTC without ETE or with mETE identified only on histopathology $(7,18)$.

In our study, we categorized four groups based on the risk of recurrence (i.e., low risk and intermediate risk w/o ETE) and the degree of ETE (i.e., minimal and gross into the strap muscles). The recurrence rates and responses to therapy were analyzed considering clinical and pathologic features related to poor prognosis: older age, male sex, unfavorable histology, multifocality, lymphovascular invasion, and lymph node metastases. At presentation, carcinomas with strap muscle invasion, gETE, included larger tumors with a higher frequency of lymphovascular invasion compared with mETE, but the frequencies of unfavorable histology or lymph node metastases were not significantly different. It is noteworthy that carcinomas with gETE had no significant difference in histopathologic features when compared with the intermediaterisk carcinomas w/o ETE.

Our data suggest that the presence of ETE and lymph node metastases are independent risk factors for recurrence based on the multivariate analysis. DFS was significantly longer in patients with low-risk carcinomas w/o ETE after adjusting for features related to poor outcomes. However, no significant difference in DFS was observed between the mETE, gETE, and intermediate risk w/o ETE groups. It should be highlighted that the low-risk group w/o ETE was less frequently treated with radioiodine. Nixon et al. (11) evaluated differentiated T1/T2 N0 thyroid carcinomas and observed a marginal effect of mETE on locoregional recurrence when compared with T1/T2 N0 carcinomas without ETE; however, in their analysis, no distinction of other intermediate-risk factors, apart from lymph node metastases, was included. A study of a small population of pediatric patients with DTC demonstrated that mETE is a strong predictor of lymph node metastases when compared to tumor size $>4 \mathrm{~cm}$ and is associated with a high frequency of persistent disease $(68 \%)$ (19). A recent meta-analysis suggested a higher risk of recurrence associated with lymph node metastases in combination with mETE. Diker-Cohen et al. (3) compared thyroid carcinomas with mETE and without ETE and found that the absolute risk of recurrence was $7 \%$ versus $6.2 \%$, respectively, while recurrence occurred in only $3.5 \%$ of N0 tumors with mETE. Indeed, our subgroup analysis demonstrates that the presence or absence of lymph node metastases in carcinomas with mETE and gETE did not result in different DFS rates.

The response to therapy is influenced by both the degree of ETE and the presence of lymph node metastases. Previous data have suggested that gross strap muscle invasion might not be distinguished from perithyroidal soft tissue extension in postoperative risk stratification (12). In the study presented here, a significant difference was observed in the response to therapy in subjects with gETE PTCs compared with the other groups. While carcinomas with mETE behaved similarly to intermediate risk w/o ETE carcinomas, carcinomas with gETE had higher rates of biochemical and/or structural incomplete responses within the first year of follow-up $(\mathrm{OR}=2.67)$, a difference that persisted at the final evaluation $(\mathrm{OR}=3.96)$. Furthermore, gETE N1 carcinomas were at the highest risk for incomplete responses within the first year $(\mathrm{OR}=5.80)$ and at the final medical visit $(\mathrm{OR}=9.32) \mathrm{com}-$ pared with mETE N0 carcinomas. Nevertheless, no difference in cancer-related survival among the groups could be identified, presumably due to a mean follow-up of less than 10 years. Similarly, reclassification of more than 2500 patients with DTC, treated at the MD Anderson Cancer Center based on the 8th edition of the AJCC staging system, reduced the number of patients categorized as T3, and the 121 patients with $\mathrm{T} 3 \mathrm{~b}$ did not present a statistically significant difference in 10-year disease-specific survival (96\%) compared with patients with T2 $(97 \%)$ or T3a (91\%) (20).

Finally, few studies have compared the recurrence rates of microcarcinomas with and without ETE, some of them evaluating N0 together with N1 tumors. A meta-analysis concluded that the presence of mETE in microcarcinomas $(\leq 1 \mathrm{~cm})$ had no significant impact on recurrence rates $(3)$. We were particularly interested in the outcomes of microcarcinomas $(\leq 1 \mathrm{~cm})$ without lymph node metastases (N0) at 
diagnosis as they could be potentially submitted to active surveillance (21). In our cohort, the presence of mETE had no independent impact on DFS or the response to therapy. However, as most patients with microcarcinomas with mETE were treated with radioiodine, we cannot exclude that the better outcome was influenced by RAI.

Our data represent a cohort of 596 patients with a PTC diagnosis from a single reference center and a similar treatment and surveillance protocol for all patients. A limitation of our study is inherent in the histological diagnosis of mETE, which can be variable and, to some extent, subjective. Moreover, the median follow-up period of less than five years might have been insufficient to evaluate differences in cancer-related survival times and to determine the impact of biochemical incomplete responses on recurrence rates. Last, radioiodine therapy was frequently used in tumors with mETE, including microcarcinomas, as they were considered intermediate risk of recurrence according to the ATA guidelines (2). Therefore, the surveillance of microcarcinomas with mETE or only surgical treatment for PTC with mETE remains uncertain. Prospective studies addressing these questions are necessary.

In summary, our data demonstrate that ETE affects recurrence rates and the response to therapy. The impact is more evident if macroscopic invasion of the strap muscle is present. The risk of recurrence attributed to ETE is independent of concomitant intermediate-risk factors, such as lymph node metastases, unfavorable histology, and lymphovascular invasion. The presence of mETE should still be considered an intermediate-risk factor for recurrence, except when it is identified in microcarcinomas without lymph node metastases. Finally, PTC with gross invasion of strap muscles should be considered an intermediate-risk factor for recurrence, and importantly, the impact on initial and long-term responses to therapy appears to be more significant in such tumors compared with other intermediate-risk carcinomas.

\section{Author Disclosure Statement}

No competing financial interests exist.

\section{Funding Information}

No funding was received.

\section{References}

1. Veiga LH, Neta G, Aschebrook-Kilfoy B, Ron E, Devesa SS 2013 Thyroid cancer incidence patterns in Sao Paulo, Brazil, and the U.S. SEER program, 1997-2008. Thyroid 23:748-757.

2. Haugen BR, Alexander EK, Bible KC, Doherty GM, Mandel SJ, Nikiforov YE, Pacini F, Randolph GW, Sawka AM, Schlumberger M, Schuff KG, Sherman SI, Sosa JA, Steward DL, Tuttle RM, Wartofsky L 20162015 American Thyroid Association Management Guidelines for Adult Patients with Thyroid Nodules and Differentiated Thyroid Cancer: The American Thyroid Association Guidelines Task Force on Thyroid Nodules and Differentiated Thyroid Cancer. Thyroid 26:1-133.

3. Diker-Cohen T, Hirsch D, Shimon I, Bachar G, Akirov A, Duskin-Bitan H, Robenshtok E 2018 Impact of minimal extra-thyroid extension in differentiated thyroid cancer: systematic review and meta-analysis. J Clin Endocrinol Metab. 103:2100-2106.
4. Nixon IJ, Wang LY, Migliacci JC, Eskander A, Campbell MJ, Aniss A, Morris L, Vaisman F, Corbo R, Momesso D, Vaisman M, Carvalho A, Learoyd D, Leslie WD, Nason RW, Kuk D, Wreesmann V, Palmer FL, Ganly I, Patel SG, Singh B, Tuttle RM, Shaha AR, Gönen M, Pathak KA, Shen WT, Sywak M, Kowalski L, Freeman J, Perrier N, Shah JP 2016 An International Multi-Institutional Validation of age 55 years as a cutoff for risk stratification in the AJCC/UICC Staging System for Well-Differentiated Thyroid Cancer. Thyroid 26:373-380.

5. Tuttle RM, Haugen B, Perrier ND 2017 Updated American Joint Committee on Cancer/tumor-node-metastasis staging system for differentiated and anaplastic thyroid cancer (eighth edition): what changed and why? Thyroid 27:751756.

6. Ahn D, Sohn JH, Jeon JH, Jeong JY 2014 Clinical impact of microscopic extrathyroidal extension in patients with papillary thyroid microcarcinoma treated with hemithyroidectomy. J Endocrinol Invest 37:167-173.

7. Hay ID, Johnson TR, Thompson GB, Sebo TJ, Reinalda MS 2016 Minimal extrathyroid extension in papillary thyroid carcinoma does not result in increased rates of either cause-specific mortality or postoperative tumor recurrence. Surgery 159:11-19.

8. Moon HJ, Kim EK, Chung WY, Yoon JH, Kwak JY 2011 Minimal extrathyroidal extension in patients with papillary thyroid microcarcinoma: is it a real prognostic factor? Ann Surg Oncol 18:1916-1923.

9. Rivera M, Ricarte-Filho J, Tuttle RM, Ganly I, Shaha A, Knauf J, Fagin J, Ghossein R 2010 Molecular, morphologic, and outcome analysis of thyroid carcinomas according to degree of extrathyroid extension. Thyroid 20: 1085-1093.

10. Chéreau N, Buffet C, Trésallet C, Tissier F, Golmard JL, Leenhardt L, Menegaux F 2014 Does extracapsular extension impact the prognosis of papillary thyroid microcarcinoma? Ann Surg Oncol 21:1659-1664.

11. Nixon IJ, Ganly I, Patel S, Palmer FL, Whitcher MM, Tuttle RM, Shaha AR, Shah JP 2011 The impact of microscopic extrathyroid extension on outcome in patients with clinical T1 and T2 well-differentiated thyroid cancer. Surgery 150: 1242-1249.

12. Amit M, Boonsripitayanon M, Goepfert RP, Tam S, Busaidy NL, Cabanillas ME, Dadu R, Varghese J, Waguespack SG, Gross ND, Graham P, Williams MD, Sturgis EM, Zafereo ME 2018 Extrathyroidal extension: does strap muscle invasion alone influence recurrence and survival in patients with differentiated thyroid cancer? Ann Surg Oncol 25:3380-3388.

13. Castagna MG, Forleo R, Maino F, Fralassi N, Barbato F, Palmitesta P, Pilli T, Capezzone M, Brilli L, Ciuoli C, Cantara S, Formichi C, Pacini F 2018 Small papillary thyroid carcinoma with minimal extrathyroidal extension should be managed as ATA low-risk tumor. J Endocrinol Invest 41:1029-1035.

14. Tam S, Amit M, Boonsripitayanon M, Busaidy NL, Cabanillas ME, Waguespack SG, Gross ND, Grubbs EG, Williams MD, Lai SY, Sturgis EM, Zafereo ME 2018 Effect of tumor size and minimal extrathyroidal extension in patients with differentiated thyroid cancer. Thyroid 28: 982-990.

15. Mete O, Rotstein L, Asa SL 2010 Controversies in thyroid pathology: thyroid capsule invasion and extrathyroidal extension. Ann Surg Oncol 17:386-391. 
16. Turk AT, Asa SL, Baloch ZW, Faquin WC, Fellegara G, Ghossein RA, Giordano TJ, LiVolsi VA, Lloyd R, Mete O, Rosai J, Suster S, Thompson LDR, Wenig BM 2019 Interobserver variability in the histopathologic assessment of extrathyroidal extension of well differentiated thyroid carcinoma supports the New American Joint Committee on Cancer Eighth Edition Criteria for Tumor Staging. Thyroid 29:619-624.

17. Ito $\mathrm{Y}$, Tomoda $\mathrm{C}$, Uruno $\mathrm{T}$, Takamura $\mathrm{Y}$, Miya A, Kobayashi K, Matsuzuka F, Kuma K, Miyauchi A 2006 Prognostic significance of extrathyroid extension of papillary thyroid carcinoma: massive but not minimal extension affects the relapse-free survival. World J Surg 30: 780-786.

18. Arora N, Turbendian HK, Scognamiglio T, Wagner PL, Goldsmith SJ, Zarnegar R, Fahey TJ 2008 Extrathyroidal extension is not all equal: implications of macroscopic versus microscopic extent in papillary thyroid carcinoma. Surgery 144:942-947; discussion 947-948.

19. Jain NK, Mostoufi-Moab S, Hawkes CP, Nelson ND, Surrey LF, Jones ZS, Adzick NS, Kazahaya K, Bauer AJ 2019 Extrathyroidal extension is an important predictor of regional lymph node metastasis in pediatric differentiated thyroid cancer. Thyroid 30:1037-1043.
20. Tam S, Boonsripitayanon M, Amit M, Fellman BM, Li Y, Busaidy NL, Cabanillas ME, Dadu R, Sherman S, Waguespack SG, Williams MD, Goepfert RP, Gross ND, Perrier ND, Sturgis EM, Zafereo ME 2018 Survival in differentiated thyroid cancer: comparing the AJCC Cancer Staging Seventh and Eighth Editions. Thyroid 28:1301-1310.

21. Ito Y, Miyauchi A, Kihara M, Higashiyama T, Kobayashi K, Miya A 2014 Patient age is significantly related to the progression of papillary microcarcinoma of the thyroid under observation. Thyroid 24:27-34.

Address correspondence to:

Debora L.S. Danilovic, MD, PhD Laboratorio de Endocrinologia Celular e Molecular (LIM25)

Faculdade de Medicina Hospital das Clinicas HCFMUSP

Universidade de Sao Paulo Avenue Dr. Arnaldo, 455, sala 4305

Sao Paulo CEP 01246-903

Brazil

E-mail: deboradanilovic@usp.br 